\title{
Novel Players in Inflammatory Bowel Disease Pathogenesis
}

\author{
Stephen F. Murphy, Ph.D., \\ Department of Medicine, University of Chicago, 900 E. 57th Street - KCBD, Chicago, IL 60637, \\ USA, Smurphy8@gmail.com
}

John H. Kwon, MD, Ph.D., and

Department of Medicine, University of Chicago, 900 E. 57th Street - KCBD, Chicago, IL 60637, USA, jkwon@medicine.bsd.uchicago.edu

David L. Boone, Ph.D.

Department of Medicine, University of Chicago, 900 E. 57th Street - KCBD, Chicago, IL 60637, USA, dboone@uchicago.edu

\begin{abstract}
Technological and conceptual advances in inflammatory bowel disease research have uncovered new mechanisms that contribute to the pathogenesis of these disorders. It is becoming increasingly clear that the microbiota of the gut and the response of intestinal cells to that microbiota can initiate or contribute to intestinal inflammation. Evidence from genetic studies have identified IBD-associated genes implicated in autophagy and innate sensing of microbes. These genes also play key roles in the homeostasis of a cell type that stands at the interface of host-microbial interaction - the Paneth cell. Here we discuss recent findings that underscore the importance of the microbiome, Paneth cells and autophagy in inflammatory bowel disease.
\end{abstract}

\section{Keywords}

Novel regulator pathways; Crohn's disease; ulcerative colitis; microbiome; Paneth cell; autophagy; NOD2; ATG16L1; IRGM; unfolded protein response; ER stress

\section{Introduction}

Inflammatory bowel disease (IBD) constitutes the chronic intestinal disorders Crohn's disease (CD) and ulcerative colitis (UC). CD can affect any area of the gastrointestinal (GI) tract and is characterised by "cobble-stone"/skipping with areas of ulceration that can be surrounded by uninflamed tissue. $\mathrm{CD}$ is associated with secondary complications such as fistulae and strictures and is microscopically identified by transmural inflammation, thickened submucosa and fissuring ulceration. Conversely, UC affects only the colon in a continuous manner and inflammation is limited to the mucosa and submucosa with cryptitis and crypt abscesses. Both diseases share complex aetiology with genetic and environmental factors conferring risk.

The current paradigm of IBD posits that initiation of the disease is due to underlying genetic polymorphisms/alterations in immune response at the level of sensing commensal and invading bacteria which is further exacerbated to disrupt signalling within the innate to

Correspondence to: David L. Boone.

Disclosure

Drs. J. Kwon and S. Murphy reported no potential conflicts of interest relevant to this article. 
adaptive immune bridge and the downstream cytokine effectors, leading to tissue damage and loss of immune homeostasis 1 . The intestine and its microbial load constitute a site of complex interactions to maintain homeostasis between a variety of cell types, invading pathogens and commensal bacteria. Here we discuss recent advances in three areas of current research in IBD which have collectively increased our understanding of the pathogenesis of IBD. Specifically, we discuss the increasingly recognized roles of the Paneth cell, microbiome and autophagy in gut inflammation and IBD.

\section{The microbiome}

IBD is thought to be initiated by a loss of balance between the microbial community and the host. Once this balance is shifted a dysbiosis in microbiome dynamics occurs that contributes to IBD pathogenesis [1-3]. The importance of a well balanced gastrointestinal microbiota is underlined by the findings in animals such as chickens, mice and rabbits that, when raised in germ-free (GF) or sterile conditions, have underdeveloped GI associated lymphoid tissue (GALT) and concomitant decreases in antibody profiles [2-5]. Specific GI bacterial commensals, such as Bacteriodes, induce host anti-microbial proteins to facilitate their own survival and resulting competitive fitness. For example, intestinal microbes induce high levels of REGIIIg expression in Paneth cells providing protection against gram positive pathogens and shaping the commensal microbiota [6,7•]. In addition to the induction of anti-microbials, the intestinal microbiota provides essential nutrients to intestinal epithelial cells, alters host metabolism and induces epithelial cell responses that maintain the intestinal barrier $[8,9]$. These microbially induced changes in the intestinal barrier can directly and indirectly alter the intestinal mucosal immune system [10-12].

It is clear that the intestinal microbiota drives the development and maintenance of the gut mucosal immune system. GF mice possess fewer Peyer's patches, a thinner lamina propria, fewer plasma cells in germinal centres and fewer lymphoid follicles, and reduced gene expression and $\mathrm{T}$ cell infiltrates. Thus, the intestinal immune system interacts with, and is shaped by, exposure to the intestinal microbiota.

The intestinal microbiota is replete with non-self antigens and adjuvants capable of activating inflammation and immune responses outside of the gut. How this detante between microbes and the mucosal immune system is maintained is not clear, but changes in the microbiota or loss of controlled immune responses to gut microbes may initiate or contribute to IBD pathogenesis. Community shifts, including dramatic reductions in the relative abundance of Firmicutes, a major phylum present in normal intestinal microbiota is observed in IBD patients [13, 14]. This decrease in Firmicutes is matched by an increase in relative abundance of proteobacteria. Also, Clostridial clusters IV and XIV exhibit relatively lower abundance in patients with IBD compared to healthy controls [14, 15]. A reduction in bacterial diversity can also be seen in inflamed regions compared to normal intestine within the same patient. It must be noted that although shifts in microbial communities may be due to an outgrowth of certain pathogenic bacteria but may, also, reflect an underlying genetic susceptibility to disease driven by, otherwise, harmless commensals. An example of this is in the dominant negative knock out (dnKO) mouse, which expresses a dominant negative, TGF $\beta$ Receptor II and is IL10 deficient, such that common Bacteroides species can invoke colitis [16••]. Such analyses of shifts in bacterial community structure and activity must be performed with caution, twin studies have identified associated microbiotic shifts in CD patients compared to control subjects but wide diversity exists even among twin controls $[17,18 \bullet \bullet]$. Such variance in microbe populations is highlighted by the lack of a so called "normal" complement of bacteria within healthy controls. 
It is not clear whether changes in the microbiome cause, or occur in response to, intestinal inflammation. However there is significant evidence that the microbiome can drive intestinal inflammation [1]. In patients, intestinal diversion, and resultant reduction in lumenal flora has been successful in amelioration of symptoms of CD.

Mouse models have provided strong evidence for the role of the microbiome in IBD. IL-10 knockout mice develop intestinal inflammation only in the presence of microbiota whereasbut GF mice with the same genetic background do not develop spontaneous colitis [19]. Transfer of GF IL-10 knockout mice to a conventional facility or gavage with intestinal microbes initiates IBD in these mice, demonstrating a causal role for the microbiome in this model. The microbiome may contribute to IBD either through modifying the intestinal metabolic profile or by activating intestinal innate immune signaling. Substantial evidence supports a role for innate immune signals, especially those driven by Toll-like receptors (TLR) in the development of IBD [20]. IL-10 knockout mice lacking the TLR signaling molecule Myd88 are protected against colitis, indicating that TLR or IL-1 receptor family signals drive the colitis in this model [21]. However, mice lacking TLR5 develop severe colitis when depleted of IL-10 which is abrogated by ablating IL-1R signaling [22]. TLR4 and TLR9 also protect against IBD in the IL10 knockout and DSS mouse models of IBD, respectively [23,24]. This is thought to be due to TLR induction of antimicrobial peptides and immunoregulatory cytokines in the gut.

Thus the microbiome can contribute bacterial ligands that either drive colitis or protect from inflammation. The complex role that the microbiota plays in IBD, exacerbating or protective, may be a reflection of the initiating stimulus for inflammation and/or the response of different intestinal cells to microbial ligands during inflammation. For example, microbial signals and TLR responses are protective in the DSS model of IBD but drive colitis in the IL-10 knockout mouse [21, 25]. This may be due to the fact that DSS colitis induces death of intestinal epithelial cells, whereas the IL-10 knockout model is driven by innate and adaptive immune cells. The microbiome may provide largely protective signals to intestinal epithelial cells while inducing inflammatory responses in the mucosal immune system. Mucosal inflammation can drive both pathogenic damaging effects in tissues, but can also provide the stimulus for imunoregulatory responses such as the activation of $\mathrm{TGFb}$ or regulatory $\mathrm{T}$ cell responses.

Since the etiology of IBD is not known, it is not currently possible to discern exactly which alterations in the microbiome play a protective or deleterious role in IBD. Despite this, some general concepts regarding the role of the microbiome in intestinal health can be posited, in decreasing order or certainty. First, IBD is a rare disease. Yet everyone has a microbiome with an ongoing immune response and a healthy GI tract. Therefore the microbiome must elicit an ongoing immune response characteristic of a healthy GI tract. It should be noted that the intestinal microbiome may play pivital roles in the pathogenesis of other autoimmune diseases outside the GI tract. Second, the microbiome promotes intestinal epithelial cell function and response to injury and therefore supports the protective functions of the intestinal epithelium, which include maintenance of the intestinal barrier, production of antimicrobial peptides and secretion of cytokines that reduce inflammation. Third, the microbiome drives innate and adaptive inflammation in the intestinal mucosa but this inflammation can be either deleterious or regulatory resulting in disease or health, respectively. Fourth, genetic studies of IBD indicate that disease pathogenesis may involve genes, including NOD2 and ATG16L1, that mediate responses to microbes, yet do not discriminate between pathogens and our commensal microbiota. As regards the last point, Paneth cells require NOD2 and ATG16L1 for their function and provide essential antimicrobial defenses in the gut, placing them at the crossroads of the microbiome and IBD genetics [26-28]. 


\section{The Paneth Cell}

Paneth cells are specialised, secretory epithelial cells found at the base of the crypt of Lieberkuhn in the small intestine. Ultrastructural studies of these cells reveal an extensive golgi and endoplasmic reticulum indicative of robust secretory capabilities. These cells are characterized by the presence of large granules high in anti-microbial protein content that are emitted into the crypt lumen and disseminate throughout the mucus layer of the intestine to prevent bacterial infection and moderate the host response to enteric commensals. Included in these proteins are the alpha-defensins, a subfamily of the defensin anti-microbial protein family and the most abundant anti-microbials of the intestine.

It is thought that secretion of these defence proteins occurs at a homeostatic level that can be further induced upon stimulation from a variety of sources including: gram negative and positive bacteria and cell wall components such as lipopolysaccharide and lipoteichoic acid. Development and differentiation of Paneth cells occurs in a tightly controlled manner mediated by WNT signalling (shown to be responsible for rapid turnover of intestinal epithelia) through its activation of the transcription factor Tcf4 and upregulation of target genes including SOX9, EPHB1 and EPHB3 [29-31]. In the context of IBD it has been shown that CD patients have significantly lower levels of TCF4 expression, which not only mediates differentiation, but also alpha-defensin production in Paneth cells [32].

Paneth cells express high levels of nucleotide-binding oligomerization domain-containing protein 2 (NOD2) [33]. NOD1 and NOD2 are cytosolic microbe-associated molecular pattern (MAMP) and danger-associated molecular pattern (DAMP) receptors that consist of a $\mathrm{C}$ terminal leucine rich repeat (LRR), responsible for recognising ligand, a central NOD domain, an $\mathrm{N}$ terminal signalling domain and one caspase activation and recruitment domain (CARD) domain [34]. Both recognise muropeptides, NOD1; meso-diaminopimelic (mesoDAP) from gram negative bacteria and NOD2; muramyl dipeptides (MDP) from all bacteria. Expression of the receptors vary; NOD1 has ubiquitious expression, whereas NOD2 is expressed only in leukocytes, dendritic cells and epithelial cells [34]. Polymorphisms have been uncovered that show association with IBD with polymorphisms in NOD1 most strongly with UC and polymorphisms in NOD2 with CD [35-37]. Many of these polymorphisms are located within the LRR domain and thus abrogate and/or attenuate recognition of their cognate ligands, e.g. the 3020insC mutation of NOD2 has diminished capacity for sensing MDP. The NOD CARD domain interacts with the CARD domain of RIP2 to initiate MAPK and NFkB signalling [34, 38, 39]. Gram-negative bacteria induce development of isolated lymphoid follicles (ILF) in the ileum and the colon via the NOD1 regulated proteins, CCL20 and alpha-defensin3 [40]. TLR2 and TLR4 can also contribute to this pathway via TNFa expression in the lamina propria. NOD1 ligands can translocate to the bone marrow to stimulate the receptor and induce neturophilic activation for enhanced bacterial killing, NOD1 KO neutrophils show reductions in such killing [41••, 42]. In terms of bacterial sensing and tolerance NOD1 deficient mice exhibit increased infection to Helicobacter species, while NOD2 show increased oral infection by Listeria [43, 44]. Thus NOD proteins are one component of the body's immune defenses against bacteria and mutations in the genes that encode these proteins are associated with IBD. This implies that the pathogenesis of IBD involves altered ability to respond to commensal or pathogenic bacteria in the gut. Paneth cells are at the front line of these defenses and are now, appropriately the subject of intense research in IBD.

In Paneth cells, NOD2 is required for production of antimicrobial peptides, loss of which results in increased bacterial burden and inflammation. NOD2 deletions or polymorphisms in NOD2 that reduce its function confer risk for CD [36, 37]. Mice deficient in NOD2 expression show altered microbial colonization, susceptibility to Listeria monocytogenes 
infection and reduced levels of alpha-defensin secretion [44]. The 1007fs SNP associated also with $\mathrm{CD}$ has also been shown to result in decreased production of antimicrobial peptides [27]. NOD2 deficiency or polymoprhisms in NOD2 also result in increased interleukin-1 production which may directly or indirectly alter Paneth cell function [45]. In addition, NOD2 deficiency in mice results in abnormal development and functioning of Peyer's patches (PPs) that does not occur immediately but rather results in larger PPs and increased numbers of $\mathrm{M}$ and CD4+ve T cells, contributing to an overall increase in levels of TNFa, IFNy, IL12 and IL4, inducing a pro-inflammatory Th1 state [46]. Thus NOD2 mutations may reduce the capacity of the mucosal immune system to control intestinal microbes while concurrently decreasing Paneth cell function and production of antimicrobial peptides, further leading to dysbiosis and intestinal inflammation.

Additional polymorphisms in CD-associated genes have been shown to distort Paneth cell function, implicating this specialised cell further in the pathogenesis of IBD, specifically of the ATG16L1 core autophagy protein (discussed below) [28]. Mice hypomorphic for ATG16L1 have altered Paneth cell gene expression and morphology, most notably an absence of the characteristic granules containing anti-microbial peptides [28]. This distinct morphology was also observed in CD patients carrying the ATG16L1 CD-associated polymorphism [28]. Thus genetic variants associated with IBD may confer risk by reducing the function of intestinal Paneth cells and leading to altered host-microbial interaction and loss of gut homeostasis.

The specialized secretory functions of Paneth cells draw attention to another pathway that has mechanistic and causal link to IBD; the UPR (unfolded protein response) induced by endoplasmic reticulum (ER) stress. ER stress is characteristically induced by accumulation of misfolded protein due to genetic abnormalities or environmental stress [47]. As a result, cells that rely on secretion for effective functioning, such as Paneth cells, are particularly dependent on a functioning UPR. The UPR is regulated by three pathways, the best characterised of which is the IRE1/XBP1 branch [47]. Activation of IRE1 by misfolded proteins in the ER allows it to activate JNK and NFkB pathways and also to act as an endoribonuclease to activate the transcription factor XBP1, leading to induction of many genes involved in the UPR [47]. A genetic locus in the vicinity of XBP1 on chromosome 22 was initially implicated with both UC and CD and subsequent candidate gene studies have confirmed this linkage [48]. Hypomorphic variants were found in IBD patients but not controls. Genetic deletion of Xbp1, specifically in the intestinal epithelium of mice, resulted in spontaneous small intestine inflammation, increased susceptibility to DSS colitis, and Paneth cell functional and phenotypic abnormalities. In the absence of XBP1, epithelium also becomes hypersensitive to cytokines and microbial ligands due to increased activation of JNK and NFkB signalling [48]. Such studies postulate that IBD may arise from genetic anomalies resulting in distorted cellular UPR as a primary driver.

Mitochondrial dysfunction and alterations in energy metabolism in general are also implicated during the onset and course of IBD. ER and mitochondria are functionally linked but the mitochondrial UPR (mtUPR), until recently, has not been investigated in terms of IBD pathogenesis. The cytoplasmic PKR receptor mediates this mtUPR via up-regulation of transcription factors EIF2a and AP-1 [49]. PKR knockout mice fail to up-regulate the chaperonin 60(C60) marker of UPR in response to DSS treatment and are somewhat protected from DSS colitis [49]. C60 and PKR are thus up-regulated in response to induced acute colitis in murine models. This may act as a new novel marker for therapy of IBD.

In summary, Paneth cells are highly specialized secretory cells that are central regulators of host microbial interaction in the gut. Genetic variants associated with IBD may have particularly important functional consequences for Paneth cells by directly altering 
antimicrobial peptide production or indirectly through the regulation of immune responses that impact Paneth cell function.

\section{Autophagy}

Variants in the core autophagy protein ATG16L1, dicussed above, have been repeatedly associated with CD [50]. Macroautophagy is a degradative cell clearance pathway responsible for engulfing long lived cellular proteins, damaged organelles and may function in immune-surveillance by clearing intracellular pathogens. A variety of inducers of this pathway have been identified, including nutrient deprivation, hypoxia and cellular infection [51]. Autophagy has been implicated as both supporting and negatively regulating tumour growth and is detrimental for correct development of embryos. ATG16L1 is responsible, in complex with ATG5-ATG12, for lipidation of microtubule-associated protein 1 light chain 3 beta (LC3B), a cytosolic protein, to the forming phagophore membrane. This conjugation to phosphoenthanolamine facilitates membrane elongation and engulfment of target protein for lysosomal mediated degradation. Recognition of targets to be degraded is controlled by ubiquitination events mediated by adaptor proteins such as p62 and NDP52 [52, 53]. The CD-associated variant of ATG16L1 results in a non-synonymous Threonine to Alanine substitution at position 300 of the multi-isoform protein, which is thought to have detrimental effects on the function of the ATG16L1 protein. Mice deficient in ATG16L1 in hematopoietic cells exhibit exacerbated IBD when exposed to DSS, decreased autophagy and increased inflammatory cytokine output (IL1B, IL18) in response to TLR ligands [54]. Other studies have shown that mice hypomorphic for ATG16L1 have distorted paneth cell morphology, activity, expression profiles and increased sensitivity to DSS treatment [28]. Increases in the number of cytosolic vesicles, decreases in the numbers of granules and modulation of lysozyme distribution in these specialised epithelial cells were also shown to occur upon decreased expression of ATG16L1. Rederivation of these mice in the context of an enhanced barrier removed these Paneth cell abnormalities, which could be recapitulated upon infection with murine norovirus (MNV) [55••]. This was mediated by the cytokines TNFa and IFN $\gamma$ and the interacting commensal bacteria, as these effects were not observed in GF mice. In a similar fashion, co-infection with MNV and Helicobacter bilis exacerbates intestinal inflammation in Mdr1a-/- mice, which lack a transmembrane transporter, required to clear toxins, expressed by IECs. Together these studies show that ATG16L1 protects against IBD potentially by controlling inflammatory cytokine production by immune cells and by controlling the function of Paneth cells. It is notable that MNV does not infect Paneth cells, suggesting that the response of ATG16L1 deficient immune cells to MNV may, indirectly, alter Paneth cells in these models.

ATG16L1 has been shown to interact and function in cooperation with NOD2 to mediate cellular responses to bacterial infection [56••]. In human DCs, autophagy directed towards S. enterica and E.coli was both NOD2 mediated and defective upon expression of the T300A polymorphism [56••-58]. ATG16L1 has been shown to be recruited to the site of bacterial entry in a NOD2 mediated manner and this association is lost upon expression of polymorphic NOD2 [56••]. Furthermore, cells from people with the ATG16L1 T300A variant appear defective in their ability to form autophagosomes around invading pathogens, indicative of a role for both ATG16L1 and NOD2 in clearance of pathogens [59]. Recent data from our lab strongly suggest that endogenous expression of T300A in human intestinal epithelia prevents bacterial entry into IECs, perhaps indicative of an autophagy independent advantage of carrying this ATG16L1 variant. Much remains to be determined regarding the exact role of the T300A polymorphism on ATG16L1's function and whether this common variant (25\% of the general populace are homozygous) may have beneficial as well as detrimental functions during host pathogen interactions. 
Numerous SNPs in another autophagy-related gene, IRGM, are associated with CD but little is known about how they contribute to pathogenesis [60]. These polymorphisms are in linkage disequilibrium with a deletion $20 \mathrm{~kb}$ upstream of IRGM that fundamentally alters expression levels differentially according to cell type [61]. IRGM contributes to intracellular clearance of microbes through autophagy [62]. Thus patients with the IRGM variant and promoter deletion may have altered handling of intracellular microbes. IRGM may also play a role in induction of apoptosis via modulation of mitochondrial function but it is not yet known if the $\mathrm{CD}$ associated mutations and/or expression levels directly affect the mitochondria [62]. IRGM has also been shown to be required for IFN $\gamma$ induced autophagy and control of murine mycobacteria infections [62]. More recently it has been shown that a synonymous $\mathrm{CD}$ associated polymorphism, the c313T variant, alters binding of the microRNA, miR-196, to IRGM mRNA and in doing so modulates expression levels impacting autophagy of invading pathogens [63•]. This is a novel example of how CDassociated polymorphisms can fundamentally alter cellular activity in a cell-type specific manner and for the first time that modulation of expression level by miRNAs can be associated with human disease risk.

\section{Conclusions}

Taken together, the microbiome, Paneth cells and autophagy are clearly important emerging players in the pathology of IBD. These are the early days of discovery and widening understanding of these players and how they interact will help to advance our knowledge of IBD. One important challenge for the future of this work is to integrate new understanding of the microbiome, Paneth cells and autophagy into the broader context of mucosal immunity, host-pathogen interactions, genetics and the pathophysiology of IBD. Ultimately, a greater understanding of the complex interactions between the microbiome and the host immune response may lead to the generation of novel approaches to the diagnosis and treatment of IBD. Assessment of the microbiome, Paneth cell function and genetics are not presently used clinically, but one can forsee a future of personalized medicine that incorporates these and other features into the clinical practice of IBD.

\section{Acknowledgments}

Dr. D. Boone has received grant support from the Crohn's and Colitis Foundation of America and the National Institutes of Health;

\section{References}

1. Sartor RB, Muehlbauer M. Microbial host interactions in IBD: implications for pathogenesis and therapy. Curr Gastroenterol Rep. 2007 Dec; 9(6):497-507. [PubMed: 18377803]

2. Thorbecke GJ, Gordon HA, Wostman B, Wagner M, Reyniers JA. Lymphoid tissue and serum gamma globulin in young germfree chickens. J Infect Dis. 1957 Nov-Dec; 101(3):237-251. [PubMed: 13491880]

3. Reyniers JA. The production and use of germ-free animals in experimental biology and medicine. Am J Vet Res. 1957 Jul; 18(68):678-687. [PubMed: 13444595]

4. Pollard M. Germfree Animals and Biological Research. Science. 1964 Jul 17; 145(3629):247-251. [PubMed: 14171565]

5. Hoffmann M, Messlik A, Kim SC, Sartor RB, Haller D. Impact of a probiotic Enterococcus faecalis in a gnotobiotic mouse model of experimental colitis. Mol Nutr Food Res. 2011 May; 55(5):703713. [PubMed: 21254393]

6. Cash HL, Whitham CV, Behrendt CL, Hooper LV. Symbiotic bacteria direct expression of an intestinal bactericidal lectin. Science. 2006 Aug 25; 313(5790):1126-1130. [PubMed: 16931762]

7. Vaishnava S, Yamamoto M, Severson KM, et al. The antibacterial lectin RegIIIgamma promotes the spatial segregation of microbiota and host in the intestine. Science. 2011 Oct 14; 334(6053):255- 
258. [PubMed: 21998396] . Demonstration that antibacterial lectin secreted from host cells maintains a microbe free zone above epithelial cells and that the maintenance of this zone promotes host microbial commensalism.

8. Hooper LV, Wong MH, Thelin A, Hansson L, Falk PG, Gordon JI. Molecular analysis of commensal host-microbial relationships in the intestine. Science. 2001 Feb 2; 291(5505):881-884. [PubMed: 11157169]

9. Xu J, Bjursell MK, Himrod J, et al. A genomic view of the human-Bacteroides thetaiotaomicron symbiosis. Science. 2003 Mar 28; 299(5615):2074-2076. [PubMed: 12663928]

10. Mazmanian SK, Liu CH, Tzianabos AO, Kasper DL. An immunomodulatory molecule of symbiotic bacteria directs maturation of the host immune system. Cell. 2005 Jul 15; 122(1):107118. [PubMed: 16009137]

11. Comstock LE, Kasper DL. Bacterial glycans: key mediators of diverse host immune responses. Cell. 2006 Sep 8; 126(5):847-850. [PubMed: 16959564]

12. Chung H, Kasper DL. Microbiota-stimulated immune mechanisms to maintain gut homeostasis. Curr Opin Immunol. 2010 Aug; 22(4):455-460. [PubMed: 20656465]

13. Kaser A, Zeissig S, Blumberg RS. Inflammatory bowel disease. Annu Rev Immunol. 2010 Mar. 28:573-621. [PubMed: 20192811]

14. Frank DN, St Amand AL, Feldman RA, Boedeker EC, Harpaz N, Pace NR. Molecularphylogenetic characterization of microbial community imbalances in human inflammatory bowel diseases. Proc Natl Acad Sci U S A. 2007 Aug 21; 104(34):13780-13785. [PubMed: 17699621]

15. Sokol H, Seksik P, Furet JP, et al. Low counts of Faecalibacterium prausnitzii in colitis microbiota. Inflamm Bowel Dis. 2009 Aug; 15(8):1183-1189. [PubMed: 19235886]

16. Bloom SM, Bijanki VN, Nava GM, et al. Commensal Bacteroides species induce colitis in hostgenotype-specific fashion in a mouse model of inflammatory bowel disease. Cell Host Microbe. 2011 May 19; 9(5):390-403. [PubMed: 21575910]. Demonstrates that a introducing a specific commensal bacterial subset can induce IBD in non-germ free mice and provides a valuable model to test host-commensal interaction and its role in IBD.

17. Dicksved J, Halfvarson J, Rosenquist M, et al. Molecular analysis of the gut microbiota of identical twins with Crohn's disease. ISME J. 2008 Jul; 2(7):716-727. [PubMed: 18401439]

18. Turnbaugh PJ, Hamady M, Yatsunenko T, et al. A core gut microbiome in obese and lean twins. Nature. 2009 Jan 22; 457(7228):480-484. [PubMed: 19043404] . This paper continues work by this group and shows that there is a core gut microbiome at the level of expressed microbial genes but not at the level of microbial species.

19. Sellon RK, Tonkonogy S, Schultz M, et al. Resident enteric bacteria are necessary for development of spontaneous colitis and immune system activation in interleukin-10-deficient mice. Infect Immun. 1998 Nov; 66(11):5224-5231. [PubMed: 9784526]

20. Cario E. Toll-like receptors in inflammatory bowel diseases: a decade later. Inflamm Bowel Dis. 2010 Sep; 16(9):1583-1597. [PubMed: 20803699]

21. Rakoff-Nahoum S, Hao L, Medzhitov R. Role of toll-like receptors in spontaneous commensaldependent colitis. Immunity. 2006 Aug; 25(2):319-329. [PubMed: 16879997]

22. Carvalho FA, Nalbantoglu I, Ortega-Fernandez S, et al. Interleukin-1\{beta\} (IL-1\{beta\}) promotes susceptibility of Toll-like receptor 5 (TLR5) deficient mice to colitis. Gut. 2011 Jun 5.

23. Matharu KS, Mizoguchi E, Cotoner CA, et al. Toll-like receptor 4-mediated regulation of spontaneous Helicobacter-dependent colitis in IL-10-deficient mice. Gastroenterology. 2009 Oct; 137(4):1380-1390. e1381-e1383. [PubMed: 19596011]

24. Katakura K, Lee J, Rachmilewitz D, Li G, Eckmann L, Raz E. Toll-like receptor 9-induced type I IFN protects mice from experimental colitis. J Clin Invest. 2005 Mar; 115(3):695-702. [PubMed: 15765149]

25. Rakoff-Nahoum S, Paglino J, Eslami-Varzaneh F, Edberg S, Medzhitov R. Recognition of commensal microflora by toll-like receptors is required for intestinal homeostasis. Cell. $2004 \mathrm{Jul}$ 23; 118(2):229-241. [PubMed: 15260992]

26. Lala S, Ogura Y, Osborne C, et al. Crohn's disease and the NOD2 gene: a role for paneth cells. Gastroenterology. 2003 Jul; 125(1):47-57. [PubMed: 12851870] 
27. Wehkamp J, Harder J, Weichenthal M, et al. NOD2 (CARD15) mutations in Crohn's disease are associated with diminished mucosal alpha-defensin expression. Gut. 2004 Nov; 53(11):16581664. [PubMed: 15479689]

28. Cadwell K, Liu JY, Brown SL, et al. A key role for autophagy and the autophagy gene Atg1611 in mouse and human intestinal Paneth cells. Nature. 2008 Nov 13; 456(7219):259-263. [PubMed: 18849966]

29. van Es JH, Jay P, Gregorieff A, et al. Wnt signalling induces maturation of Paneth cells in intestinal crypts. Nat Cell Biol. 2005 Apr; 7(4):381-386. [PubMed: 15778706]

30. Bastide P, Darido C, Pannequin J, et al. Sox9 regulates cell proliferation and is required for Paneth cell differentiation in the intestinal epithelium. J Cell Biol. 2007 Aug 13; 178(4):635-648. [PubMed: 17698607]

31. Batlle E, Henderson JT, Beghtel H, et al. Beta-catenin and TCF mediate cell positioning in the intestinal epithelium by controlling the expression of EphB/ephrinB. Cell. 2002 Oct 18; 111(2): 251-263. [PubMed: 12408869]

32. Wehkamp J, Wang G, Kubler I, et al. The Paneth cell alpha-defensin deficiency of ileal Crohn's disease is linked to Wnt/Tcf-4. J Immunol. 2007 Sep 1; 179(5):3109-3118. [PubMed: 17709525]

33. Ogura Y, Lala S, Xin W, et al. Expression of NOD2 in Paneth cells: a possible link to Crohn's ileitis. Gut. 2003 Nov; 52(11):1591-1597. [PubMed: 14570728]

34. Franchi L, Warner N, Viani K, Nunez G. Function of Nod-like receptors in microbial recognition and host defense. Immunol Rev. 2009 Jan; 227(1):106-128. [PubMed: 19120480]

35. McGovern DP, Hysi P, Ahmad T, et al. Association between a complex insertion/deletion polymorphism in NOD1 (CARD4) and susceptibility to inflammatory bowel disease. Hum Mol Genet. 2005 May 15; 14(10):1245-1250. [PubMed: 15790594]

36. Ogura Y, Bonen DK, Inohara N, et al. A frameshift mutation in NOD2 associated with susceptibility to Crohn's disease. Nature. 2001 May 31; 411(6837):603-606. [PubMed: 11385577]

37. Hugot JP, Chamaillard M, Zouali H, et al. Association of NOD2 leucine-rich repeat variants with susceptibility to Crohn's disease. Nature. 2001 May 31; 411(6837):599-603. [PubMed: 11385576]

38. Kobayashi K, Inohara N, Hernandez LD, et al. RICK/Rip2/CARDIAK mediates signalling for receptors of the innate and adaptive immune systems. Nature. 2002 Mar 14; 416(6877):194-199. [PubMed: 11894098]

39. Chin AI, Dempsey PW, Bruhn K, Miller JF, Xu Y, Cheng G. Involvement of receptor-interacting protein 2 in innate and adaptive immune responses. Nature. 2002 Mar 14; 416(6877):190-194. [PubMed: 11894097]

40. Bouskra D, Brezillon C, Berard M, et al. Lymphoid tissue genesis induced by commensals through NOD1 regulates intestinal homeostasis. Nature. 2008 Nov 27; 456(7221):507-510. [PubMed: 18987631]

41. Clarke TB, Davis KM, Lysenko ES, Zhou AY, Yu Y, Weiser JN. Recognition of peptidoglycan from the microbiota by Nod1 enhances systemic innate immunity. Nat Med. 2010 Feb; 16(2):228231. [PubMed: 20081863] . This paper indicates that gut microbial products, in this case NOD1 ligands derived from peptidoglycan, alter systemic innate immunity by entering the systemic circulation from the gut.

42. Masumoto J, Yang K, Varambally S, et al. Nod1 acts as an intracellular receptor to stimulate chemokine production and neutrophil recruitment in vivo. J Exp Med. 2006 Jan 23; 203(1):203213. [PubMed: 16418393]

43. Viala J, Chaput C, Boneca IG, et al. Nod1 responds to peptidoglycan delivered by the Helicobacter pylori cag pathogenicity island. Nat Immunol. 2004 Nov; 5(11):1166-1174. [PubMed: 15489856]

44. Kobayashi KS, Chamaillard M, Ogura Y, et al. Nod2-dependent regulation of innate and adaptive immunity in the intestinal tract. Science. 2005 Feb 4; 307(5710):731-734. [PubMed: 15692051]

45. Maeda S, Hsu LC, Liu H, et al. Nod2 mutation in Crohn's disease potentiates NF-kappaB activity and IL-1beta processing. Science. 2005 Feb 4; 307(5710):734-738. [PubMed: 15692052]

46. Barreau F, Meinzer U, Chareyre F, et al. CARD15/NOD2 is required for Peyer's patches homeostasis in mice. PLoS One. 2007; 2(6):e523. [PubMed: 17565376]

47. Walter P, Ron D. The unfolded protein response: from stress pathway to homeostatic regulation. Science. 2011 Nov 25; 334(6059):1081-1086. [PubMed: 22116877] 
48. Kaser A, Lee AH, Franke A, et al. XBP1 links ER stress to intestinal inflammation and confers genetic risk for human inflammatory bowel disease. Cell. 2008 Sep 5; 134(5):743-756. [PubMed: 18775308]

49. Rath E, Berger E, Messlik A, et al. Induction of dsRNA-activated protein kinase links mitochondrial unfolded protein response to the pathogenesis of intestinal inflammation. Gut. 2011 Oct 13.

50. Hampe J, Franke A, Rosenstiel P, et al. A genome-wide association scan of nonsynonymous SNPs identifies a susceptibility variant for Crohn disease in ATG16L1. Nat Genet. 2007 Feb; 39(2):207211. [PubMed: 17200669]

51. Levine B, Deretic V. Unveiling the roles of autophagy in innate and adaptive immunity. Nat Rev Immunol. 2007 Oct; 7(10):767-777. [PubMed: 17767194]

52. Bjorkoy G, Lamark T, Brech A, et al. p62/SQSTM1 forms protein aggregates degraded by autophagy and has a protective effect on huntingtin-induced cell death. J Cell Biol. 2005 Nov 21; 171(4):603-614. [PubMed: 16286508]

53. Thurston TL, Ryzhakov G, Bloor S, von Muhlinen N, Randow F. The TBK1 adaptor and autophagy receptor NDP52 restricts the proliferation of ubiquitin-coated bacteria. Nat Immunol. 2009 Nov; 10(11):1215-1221. [PubMed: 19820708]

54. Saitoh T, Fujita N, Jang MH, et al. Loss of the autophagy protein Atg16L1 enhances endotoxininduced IL-1beta production. Nature. 2008 Nov 13; 456(7219):264-268. [PubMed: 18849965]

55. Cadwell K, Patel KK, Maloney NS, et al. Virus-plus-susceptibility gene interaction determines Crohn's disease gene Atg16L1 phenotypes in intestine. Cell. 2010 Jun 25; 141(7):1135-1145. [PubMed: 20602997] . Builds on previous work by these authors showing that ATG16L1 is important for Paneth cell morphology and function. In this study they demonstrate that a specific murine norovirus is required to initiate the Paneth cell phenotypes seen in ATG16L1 hypomorphic mice.

56. Travassos LH, Carneiro LA, Ramjeet M, et al. Nod1 and Nod 2 direct autophagy by recruiting ATG16L1 to the plasma membrane at the site of bacterial entry. Nat Immunol. 2010 Jan; 11(1): 55-62. [PubMed: 19898471] . This study functionally links two important CD-associated genes, ATG16L1 and NOD2, by showing that proteins interact at the site of microbial entry into cells.

57. Cooney R, Baker J, Brain O, et al. NOD2 stimulation induces autophagy in dendritic cells influencing bacterial handling and antigen presentation. Nat Med. $2010 \mathrm{Jan} ; 16(1): 90-97$. [PubMed: 19966812]

58. Homer CR, Richmond AL, Rebert NA, Achkar JP, McDonald C. ATG16L1 and NOD2 interact in an autophagy-dependent antibacterial pathway implicated in Crohn's disease pathogenesis. Gastroenterology. 2010 Nov; 139(5):1630-1641. 1641, e1631-e1632. [PubMed: 20637199]

59. Kuballa P, Huett A, Rioux JD, Daly MJ, Xavier RJ. Impaired autophagy of an intracellular pathogen induced by a Crohn's disease associated ATG16L1 variant. PLoS One. 2008; 3(10):e3391. [PubMed: 18852889]

60. Parkes M, Barrett JC, Prescott NJ, et al. Sequence variants in the autophagy gene IRGM and multiple other replicating loci contribute to Crohn's disease susceptibility. Nat Genet. 2007 Jul; 39(7):830-832. [PubMed: 17554261]

61. McCarroll SA, Huett A, Kuballa P, et al. Deletion polymorphism upstream of IRGM associated with altered IRGM expression and Crohn's disease. Nat Genet. 2008 Sep; 40(9):1107-1112. [PubMed: 19165925]

62. Singh SB, Davis AS, Taylor GA, Deretic V. Human IRGM induces autophagy to eliminate intracellular mycobacteria. Science. 2006 Sep 8; 313(5792):1438-1441. [PubMed: 16888103]

63. Brest P, Lapaquette P, Souidi M, et al. A synonymous variant in IRGM alters a binding site for miR-196 and causes deregulation of IRGM-dependent xenophagy in Crohn's disease. Nat Genet. 2011 Mar; 43(3):242-245. [PubMed: 21278745] . This paper shows that a CD-associated genetic variant that does not change the amino acid sequence of a protein can still have effects of gene function. In this case they show that a non-synonymous variant in IRGM changes a miRNA binding site and reduces IRGM message stability. 